\title{
A stochastic model depending on the infection rate :COVID19 case in Djibouti
}

\author{
Liban ISMAIL* \\ Laboratoire de Mathématiques et Informatique (LMI) \\ Université de Djibouti, Campus Balbala \\ Croisement RN2-RN5, $B P=1904$
}

\begin{abstract}
.
The novel Coronavirus (COVID-19) is spreading and has caused a large-scale infection in China since December 2019. The first infected person was declared on March 18, 2020 in Djibouti. This has led to a significant impact on the lives and economy in Djibouti and other countries.
\end{abstract}

In this study, we propose a double compartment stochastic model which describes the evolution of the infection rate and the evolution of the number of infected in the period from May 20 to June 23, 2020. We will also propose the evolution of people infected in two states, recovered and deceased.

Keywords: Stochastic model epidemic; COVID-19; Simulation.

2010 Mathematics Subject Classification: 60H10, 65C20.

\section{Introduction}

On 31 December 2019, WHO was informed of cases of pneumonia of unknown cause in Wuhan City, China. A novel coronavirus was identified as the cause by Chinese authorities on 7 January 2020 and was temporarily named "2019-nCoV".

Coronaviruses $(\mathrm{CoV})$ are a large family of viruses that cause illness ranging from the common cold to more severe diseases. A novel coronavirus ( $\mathrm{nCoV}$ ) is a new strain that has not been previously identified in humans. The new virus was subsequently named the "COVID-19 virus".

\footnotetext{
*Correponding author: Liban ISMAIL (email: liban_ismail_abdillahi@univ.edu.dj)
} 
On 11 March 2020, the rapid increase in the number of cases outside China led WHO(World Health Organization) Director-General Dr Tedros Adhanom Ghebreyesus to announce that the outbreak could be characterized as a pandemic. By then more than 118000 cases had been reported in 114 countries, and 4291 deaths had been recorded.

By mid-March 2020, the WHO European Region had become the epicentre of the epidemic, reporting over 40 percent of globally confirmed cases. As of 28 April 2020, 63 percent of global mortality from the virus was from the Region.

It is since March 18 that the Republic of Djibouti declared its first case of COVID-19 and recorded a very low mortality rate. The authorities imposed general confinement, with the exception of essential services. Traffic has been kept to a minimum, and requires administrative clearance.

In this study, we propose a stochastic epidemiological model which describes the evolution of the transmission rate and the number of infected. First, we propose a double compartment stochastic model and secondly we will propose a system of three stochastic differential equations depending on the infection rate.

In the following section, we will discuss our mathematical model and formulation of the model elaborately.

\section{Model}

In this paper, we present a model to study the evolution of the number of infected individuals by the coronavirus (COVID-19). Let $I_{t}$ the number of infected individuals at day $t$ and $\beta(t)$ the rate infection at day $t$. It is common, see for instance [4] and [5], to consider that this number is driven by a Stochastic Dierential Equation (SDE) of the form

$$
\left\{\begin{array}{l}
d \beta_{t}=\mu \beta_{t} d t+\sigma_{0} \beta_{t} d B_{t} \\
d I_{t}=\beta_{t} I_{t} d t+\sigma_{1} I_{t} d B_{t}
\end{array}\right.
$$

The first equation of the above system is describing the infection rate dynamics with $\mu$ as a variation rate and as a random error intensity $\sigma_{0}>0$ which we suppose here governed by a Brownian Process (BP) $B_{t}$. The second equation describes infected individuals number dynamics, but this time with variable infection rate, that is nothing else than the solution of the first SDE. The parameter $\sigma_{1}$ denotes the corresponding random error intensity. 


\section{Data and parameter estimation}

In this study, we considered the country Djibouti less infected with the COVID-19 virus and collected the data available online in chronological order from Worldometer $[1,3]$.

We have estimated the important model parameters using the Djibouti infection cases from 20th Mai to 23th June, 2020. To estimate the important model parameters we using the properties of Ito and Brownian motion from the real data source.

To parameterize equation 1 , we use a discretization and then a passage to average in the first equation of 1 , then we get the following approximation

$$
\mathbb{E}\left(\Delta \beta_{t}\right) \approx \mu \mathbb{E}\left(\beta_{t}\right) \Delta_{t}
$$

SO

$$
\mu \approx \frac{\mathbb{E}\left(\Delta \beta_{t}\right)}{\mathbb{E}\left(\beta_{t}\right) \Delta_{t}}
$$

where $\mathbb{E}(X)$ represent the empirical mean of $X$ and $\Delta t$ is to choose.

To obtain an estimate of intensity error, we apply the variance in the two equations and we find:

$$
\begin{aligned}
& \mathbb{V}\left(\Delta \beta_{t}\right) \approx \sigma_{0}^{2} \mathbb{V}\left(\beta_{t}\right) \Delta_{t}, \\
& \mathbb{V}\left(\Delta I_{t}\right) \approx \sigma_{1}^{2} \mathbb{V}\left(I_{t}\right) \Delta_{t},
\end{aligned}
$$

where $\mathbb{V}(X)$ represent the variance of $X$. So we have,

$$
\begin{gathered}
\sigma_{0} \approx \sqrt{\frac{\mathbb{V}\left(\Delta \beta_{t}\right)}{\mathbb{V}\left(\beta_{t}\right) \Delta_{t}}} \\
\sigma_{1} \approx \sqrt{\frac{\mathbb{V}\left(\Delta I_{t}\right)}{\mathbb{V}\left(I_{t}\right) \Delta_{t}}}
\end{gathered}
$$

\begin{tabular}{|l|l|}
\hline \multicolumn{2}{|l|}{ Table 1: Parameters estimation for Djibouti. } \\
\hline Parameter & value:20 Mai-22 June \\
\hline$\mu$ & 0.06 \\
$\sigma_{0}$ & 0.21948 \\
$\sigma_{1}$ & 1.20 \\
\hline
\end{tabular}




\section{Dynamics of the infected number and the infec- tion rate}

In this section, we will simulate the dynamic between the number of infected and the infection rate.

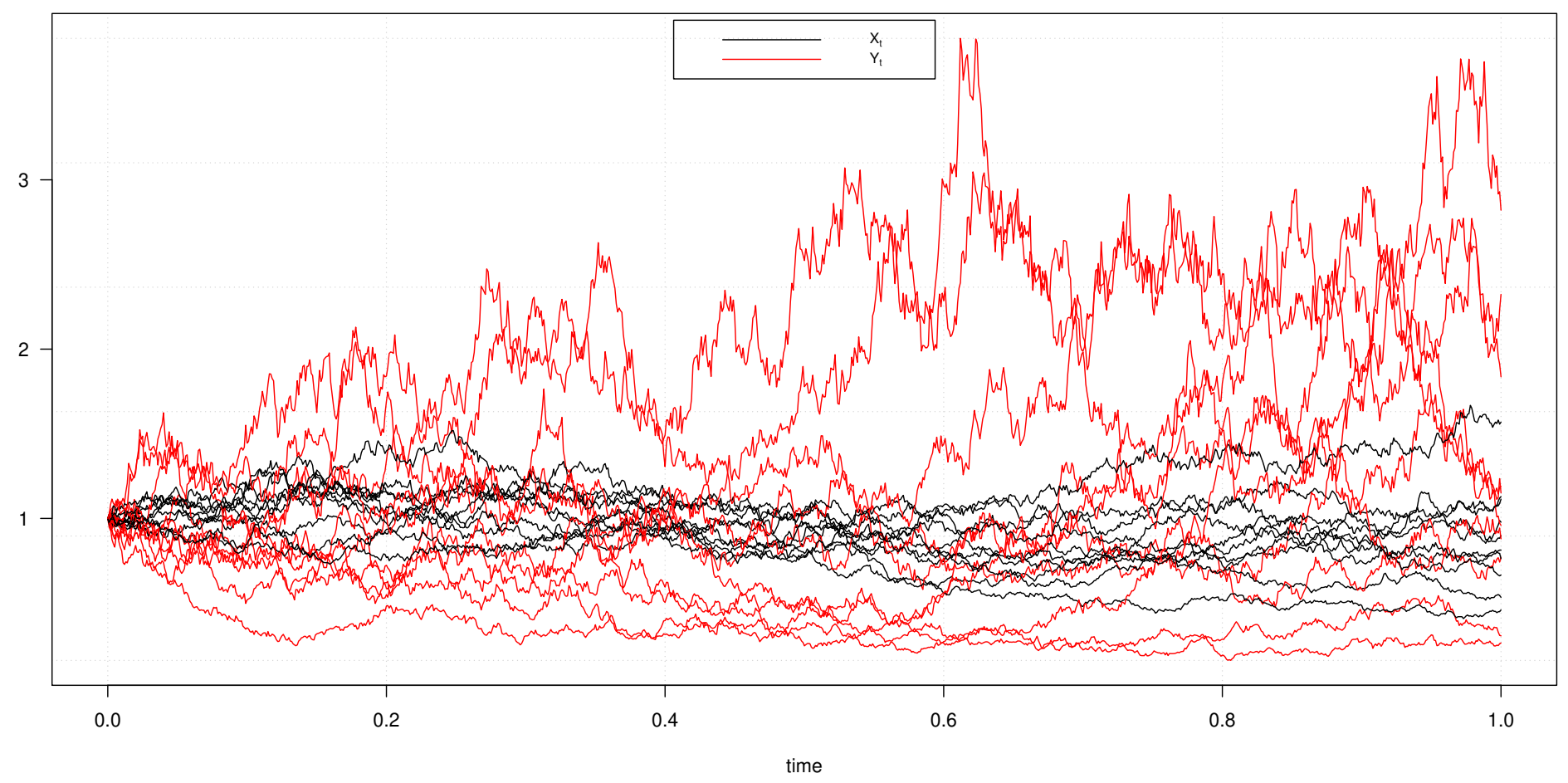

Figure 1: The evolution of the number of infected and the infection rate over time.

The $X_{i}$ represent the rate of variation of infection and the $Y_{i}$ represent the variation of infections. For the date scale, 0.2 represents 6 days.

With 10 simulations, we notice that the variation of infected varies according to the variation of the infection rate.

In Figure 2, for a $\mu=0.5$, we note that the number of infected increases to 10 . In Figure 3, for a $\mu=2$, we note that the number of infected increases to 200 .

The higher the value of the infection rate $\beta_{t}$, the stronger the spread of the virus in the population.

When the value of $\beta_{t}$ increases, the transmission of the virus in the population is important, the number of infected will increase and there will be an increase in the recovered and the deceased. In Figures 2 and 3, we also see that the spread of the virus will continue to change over time in a significant way. 


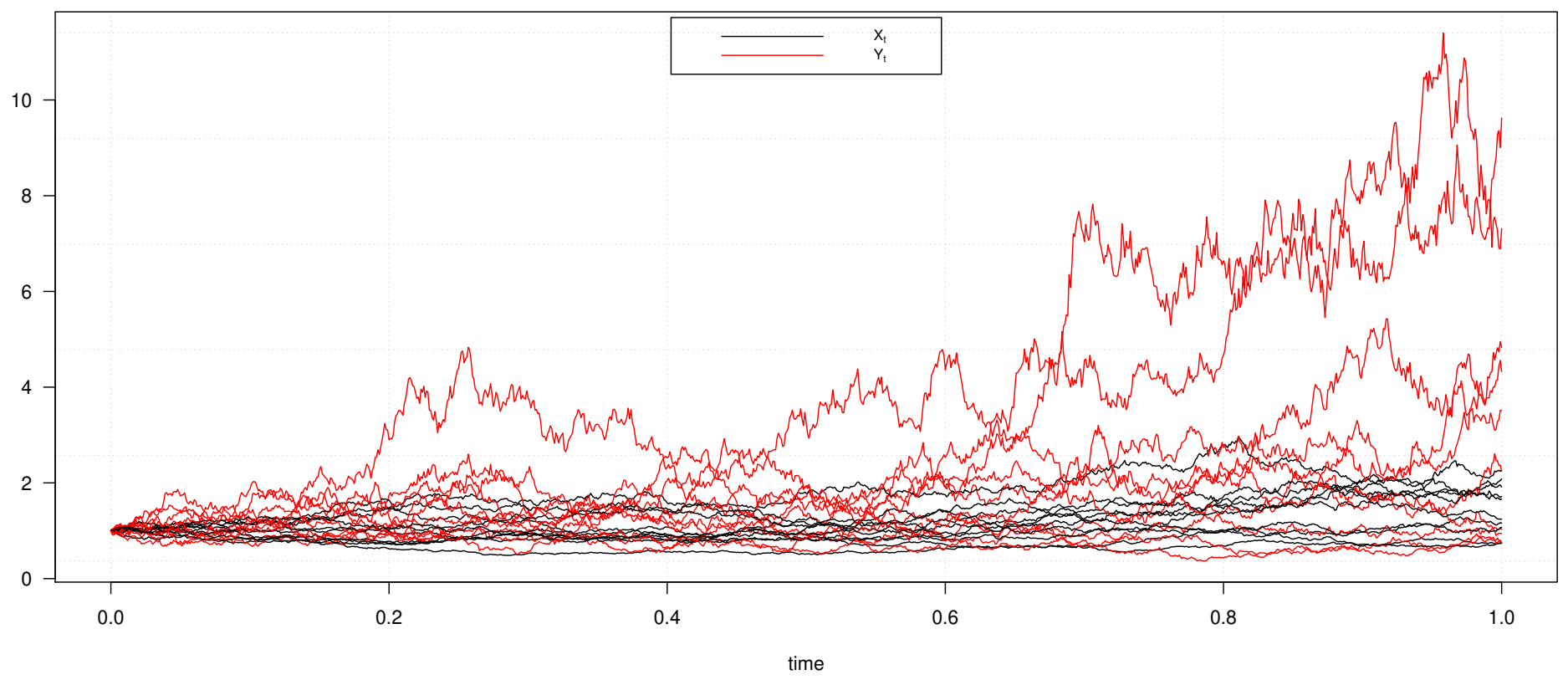

Figure 2: The evolution of the number of infected and the infection rate over time with $\mu=0.5$.

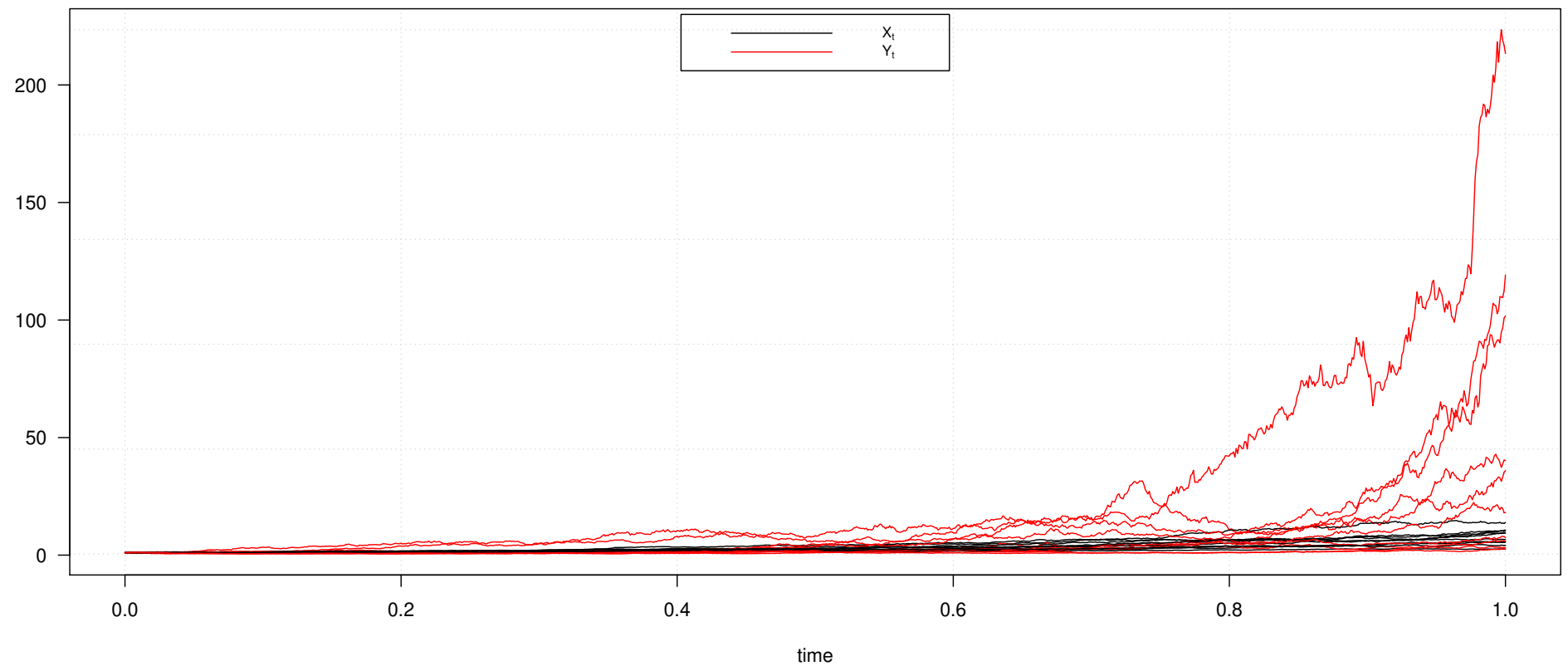

Figure 3: The evolution of the number of infected and the infection rate over time with $\mu=2$. 


\section{Dynamics of recovered and death}

In this section, we will study the dynamics of those infected, cured and dead, knowing that all three depend on the infection rate $\beta$. For that we need first to divide infected individuals into two states, the recovered and deceased. If we denote respectively by $R_{t}$ and $D_{t}$ the recovered and deceased respectively.

We fix the infection rate $\beta_{t}=\bar{\beta}$ its empirical mean.

$$
\left\{\begin{array}{l}
d I_{t}=\bar{\beta} I_{t} d t+\sigma_{1} I_{t} d B_{t} \\
d R_{t}=\beta_{1} \bar{\beta} I_{t} d t+\sigma_{2} R_{t} d B_{t} \\
d D_{t}=\beta_{2} \bar{\beta} d t+\sigma_{3} D_{t} d B_{t}
\end{array}\right.
$$

where

$$
\begin{aligned}
\beta_{1} & =\frac{\mathbb{E}\left(\Delta R_{t}\right)}{\mathbb{E}\left(I_{t}\right)} \cdot \frac{1}{\Delta t} \\
\beta_{2} & =\frac{\mathbb{E}\left(\Delta D_{t}\right)}{\mathbb{E}\left(I_{t}\right)} \cdot \frac{1}{\Delta t} \\
\sigma_{1} & =\sqrt{\frac{\mathbb{V}\left(\Delta I_{t}\right)}{\mathbb{V}\left(I_{t}\right)} \cdot \frac{1}{\Delta t}} \\
\sigma_{2} & =\sqrt{\frac{\mathbb{V}\left(\Delta R_{t}\right)}{\mathbb{V}\left(R_{t}\right)} \cdot \frac{1}{\Delta t}} \\
\sigma_{3} & =\sqrt{\frac{\mathbb{V}\left(\Delta D_{t}\right)}{\mathbb{V}\left(D_{t}\right)} \cdot \frac{1}{\Delta t}}
\end{aligned}
$$

\begin{tabular}{|l|l|}
\hline \multicolumn{2}{|c|}{ Table 2: Parameters estimation. } \\
\hline Parameter & value:20 Mai-23 June \\
\hline $\bar{\beta}$ & 0.1021 \\
$\sigma_{0}$ & 0.21948 \\
$\beta_{1}$ & 0.0063 \\
$\beta_{2}$ & 0.0007 \\
$\sigma_{1}$ & 1.20 \\
$\sigma_{2}$ & 1.1 \\
$\sigma_{3}$ & 1.29 \\
\hline
\end{tabular}

In the following figures, the $X_{i}$ represent the variation of number infected, the $Y_{i}$ represent the variation of recovered and $Z_{i}$ represent the variation of deceased. For the date scale, 0.2 represents 6 days. 


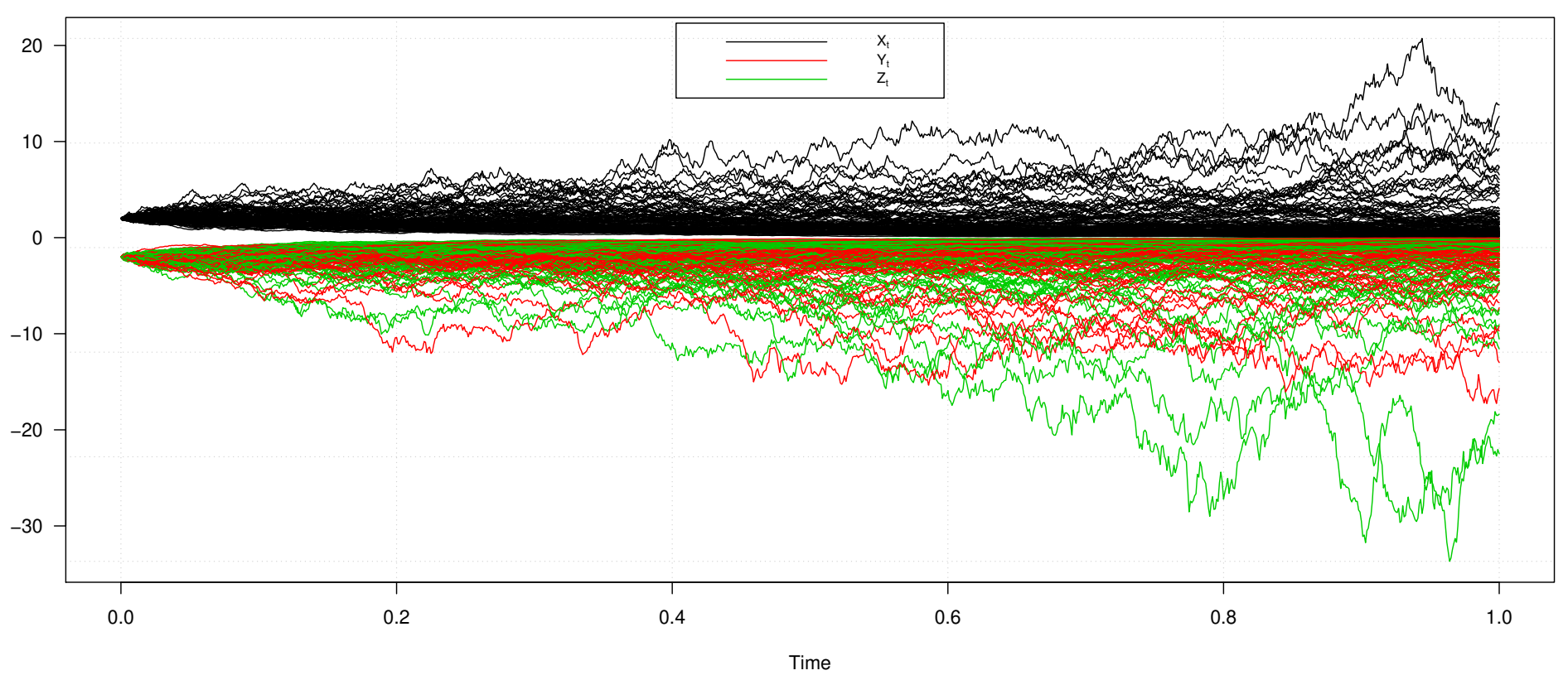

Figure 4: The evolution of the number of infected, the recovered and the deceased individuals over time.

In figures 4 , we note that the evolution over time of the infected, the cured and the deceased are proportional, the more we have the infected and the more we will be cured and died.

In Figure 5, the system dynamics show that the evolution of the model follows a centered normal law. The proportionality between the number of infected on one side and the number of cured and deaths on the other side is proven by the marginal densities. 


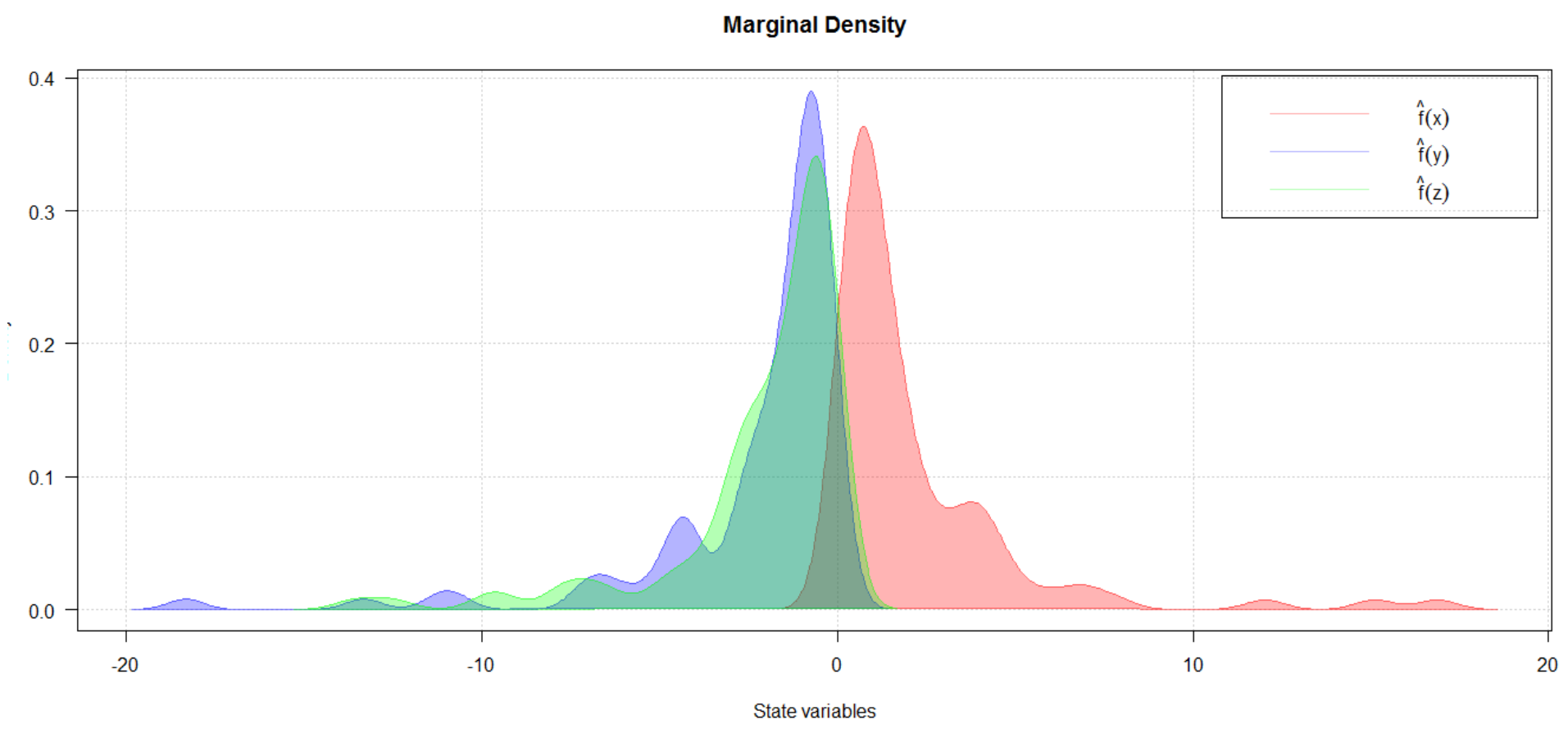

Figure 5: The marginal density of the number of infected, the recovered and the deceased individuals over time.

Now if we vary the value of $\bar{\beta}$, we take two values for $\bar{\beta}=0.5$ and $\bar{\beta}=2$, we see that figures 6 and 7 .

We note that if we increase $\bar{\beta}$ we will automatically have an increase in the numbers of infected and the numbers of people cured and deceased. In fugures 7 , we also note that over time we will have more people (cured or deceased) than infected people. 


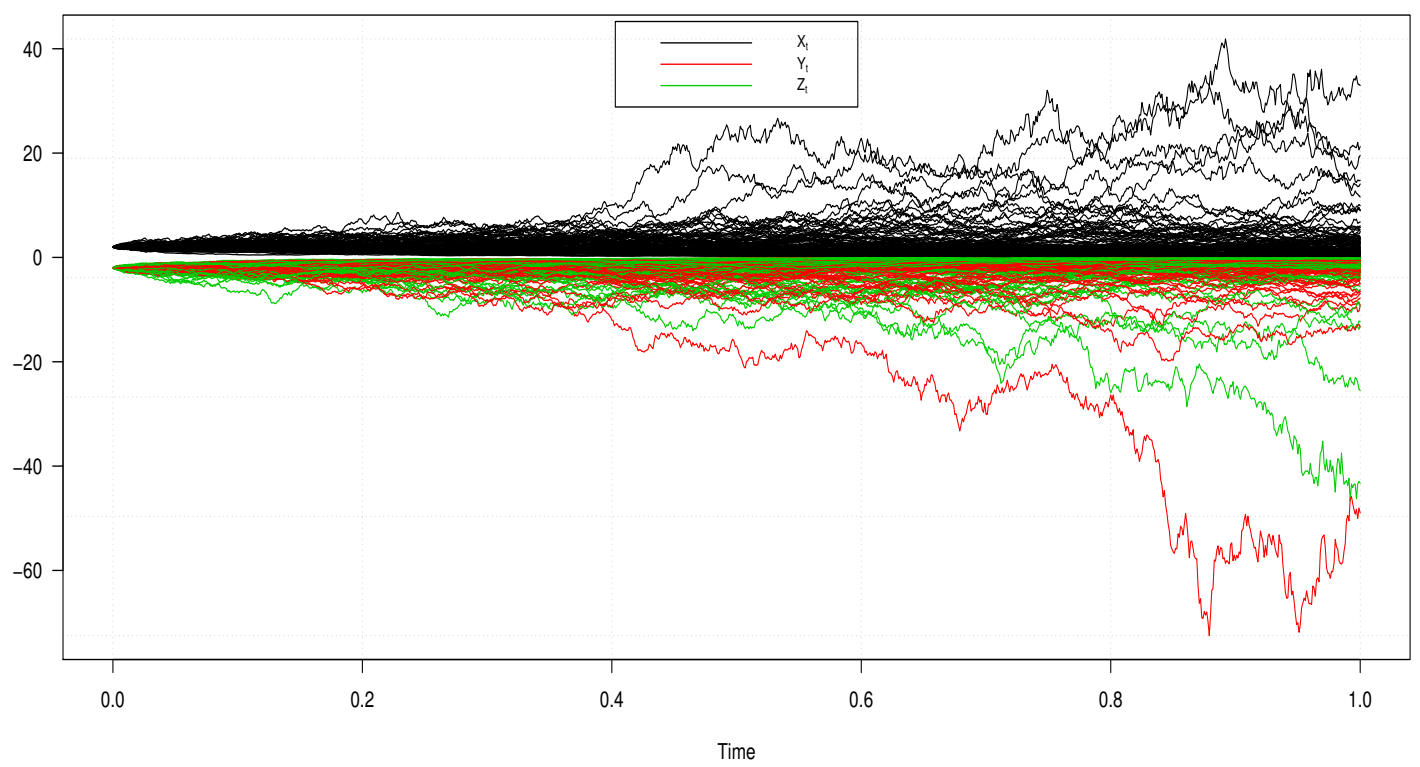

(a)

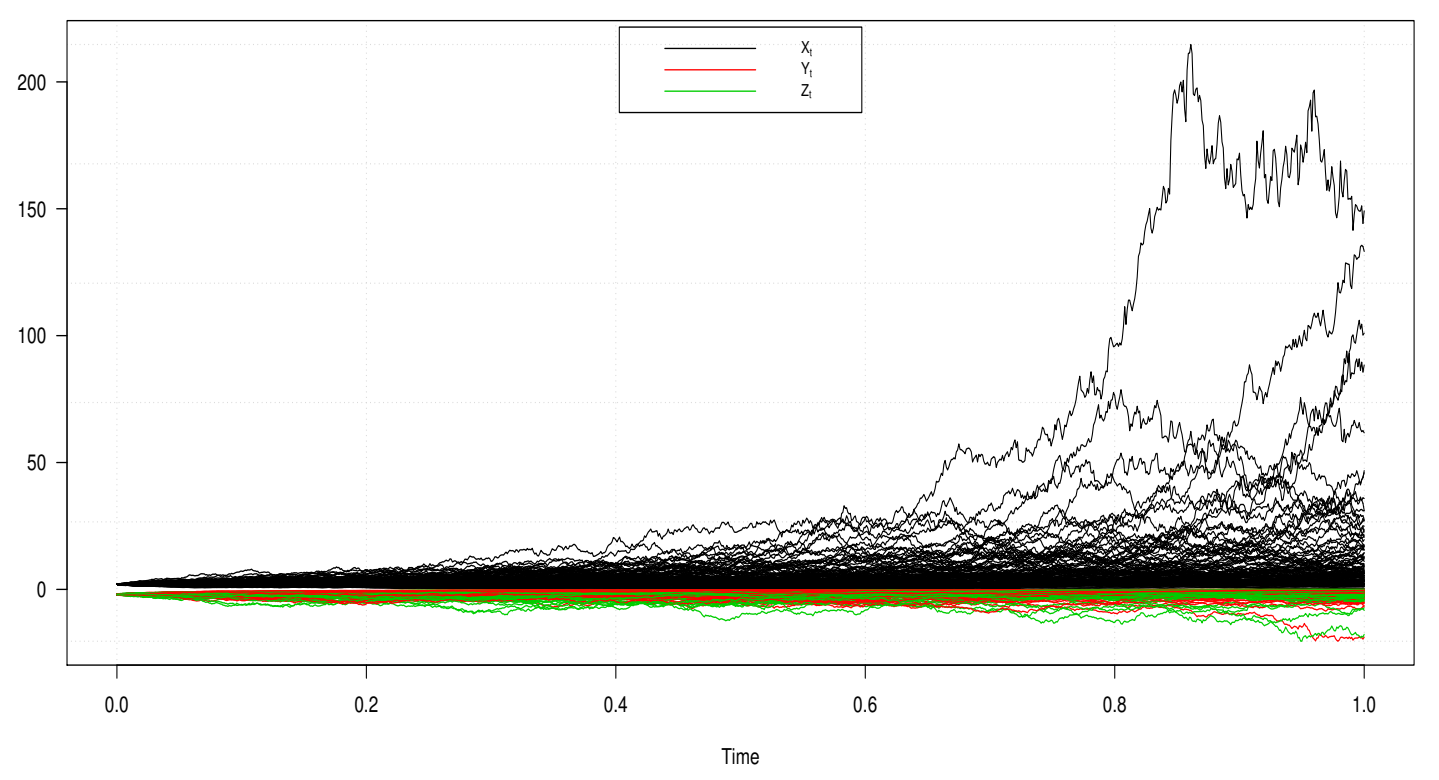

(b)

Figure 6: (a)Dynamic of the model 8 with $\bar{\beta}=0.5$ from May 20 to June 23, 2020 (b) Dynamic of the model 8 with $\bar{\beta}=2$ from May 20 to June 23, 2020 


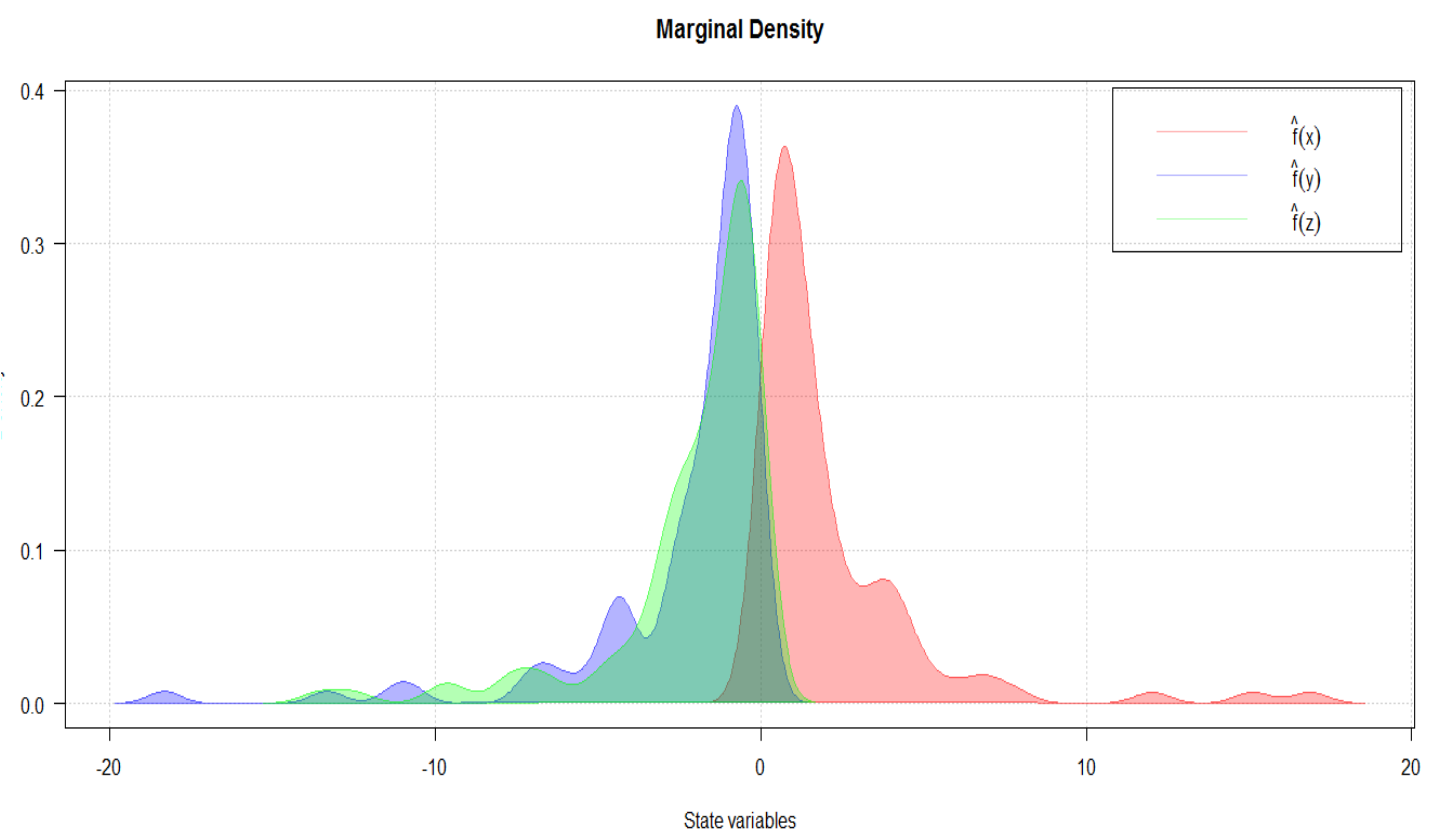

(a)

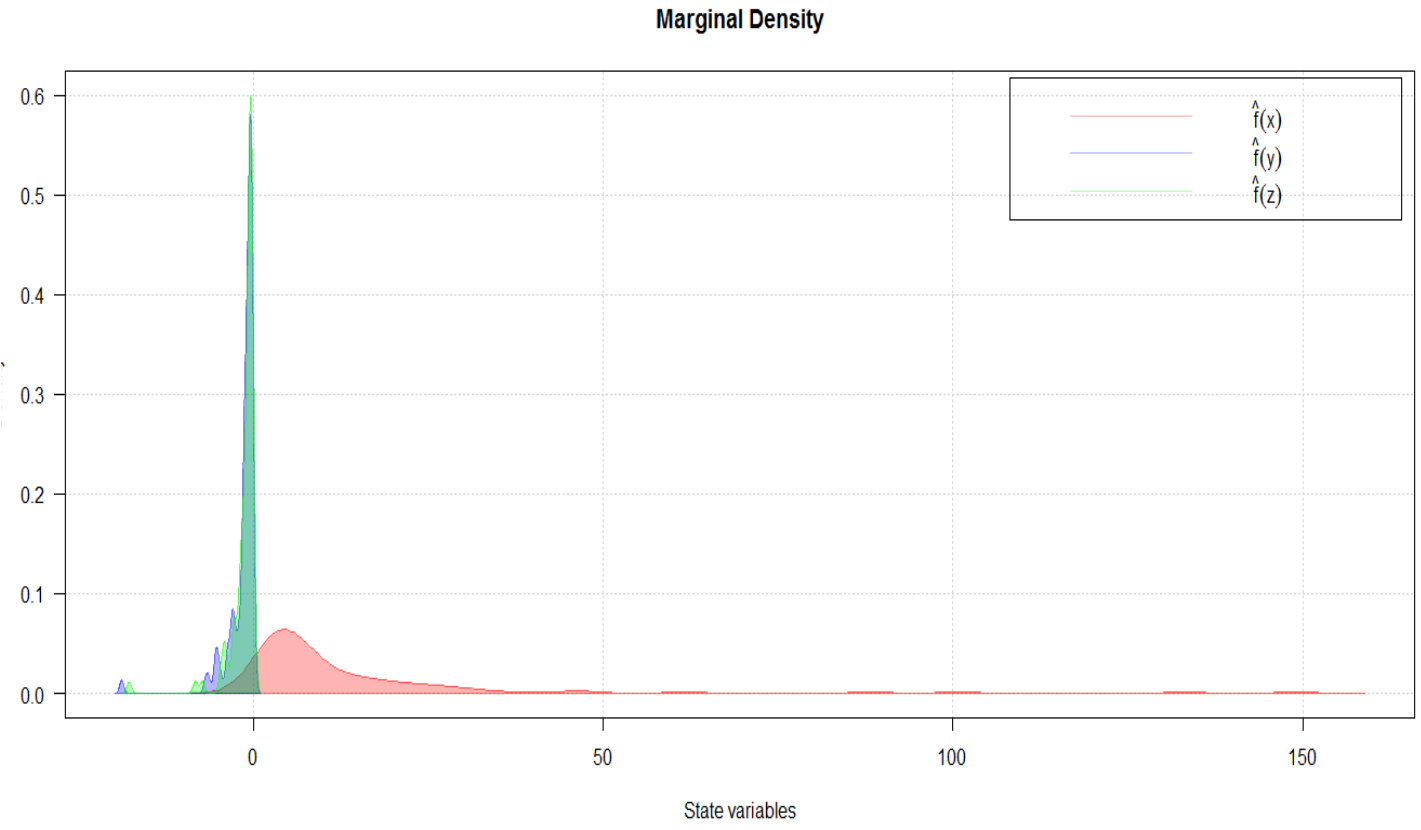

(b)

Figure 7: (a)The marginal density of the model 8 with $\bar{\beta}=0.5$ from May 20 to June 23, 2020 (b) The marginal density of the model 8 with $\bar{\beta}=2$ from May 20 to June 23,2020 


\section{Conclusion}

In this study, we have formulated a stochastic epidemic model for pandemic COVID19 depending the infection rate. In conclusion, our simulation shows that the infection rate is a factor that influences the control of COVID-19. In our simulations we found that the compartments (infected, recovered, deceased) depend on the infection rate. The infection rate is the key factor influencing the pandemic.

When there is a sign of an epidemic, we need to educate people and take all the possible protective measures, such as wearing a mask, respecting barrier gestures, respecting distance and staying indoors to reduce the risk of infection. Although the number of new infections is decreasing, there is always the possibility of future outbreaks if there are no adequate protections or if people return to work earlier. The public must not relax its vigilance against the transmission of this highly contagious disease.

\section{References}

[1] https://www.worldometers.info/coronavirus/

[2] https://en.wikipedia.org/wiki/Djibouti

[3] https://www.who.int/emergencies/diseases/novel-coronavirus-2019

[4] Tom Britton, Etienne Pardoux and Frank Ball: Stochastic Epidemic Models with Inference. Lecture Notes in Mathematics 2255 1st ed. (2019).

[5] M. Choisy, 1,2 J.-F. Guegan, 2 and P. Rohani 1,3: Mathematical Modeling of Infec- tious Diseases Dynamics. Encyclopedia of Infectious Diseases: Modern Methodologies. John Wiley Sons, Inc. (2007).

[6] P. Lekone, B. Finkenst“adt, Statistical Inference in a Stochastic Epidemic SEIR Model with Control Intervention: Ebola as a Case Study, Biometrics, 62 (2006), 1170-1177.

[7] A. Rezgui, Stochastic Model for the dynamics of Infected Individuals Number by COVID-19, Preprint April 2020 DOI: 10.13140/RG.2.2.23905.04968 